\title{
Pattern Formation in Competition-Diffusion Systems in Nonconvex Domains
}

By

\author{
Hiroshi Matano* and Masayasu Mimura*
}

\begin{abstract}
We deal with a weakly-coupled system of semilinear parabolic equations, namely a competition-diffusion system, and prove the existence of a stable spatially-inhomogeneous equilibrium solution on the assumption that the spatial domain is far from being convex and that the corresponding system of ODEs in the absence of diffusion posesses at least two distinct asymptotically stable equilibria. We also consider a non-weakly coupled system of competition type involving cross-diffusion terms, which leave the system quasilinear but no longer semilinear. The point of interest is to see how the shape of the spatial domain or the presence of cross-diffusion terms contributes to the occurrence of pattern formation.
\end{abstract}

\section{§1. Introduction}

The primary concern of this paper is the study of a certain class of quasilinear parabolic systems - more precisely, the so-called competition-diffusion systems, which arise in various fields of sciences and have long been the subject of extensive mathematical studies.

One of the topics of interest is the problem of "spatial pattern formation", by which we mean the convergence of solutions to some stable spatially-inhomogeneous pattern as time tends to infinity. The term "pattern" refers, in the present context, to either an equilibrium solution or a periodic solution. Thus our basic concern is to find, if any, a spatially-inhomogeneous equilibrium (or periodic) solution that is stable in a certain sense. Once such an equilibrium or periodic solution is found, the next problem to arise is to figure out the domain of attraction of this equilibrium (or periodic) solution so that we may know what sort of initial data lead to the specific spatial pattern and what sort not; but this question will not be pursued in the present paper.

Recieved May 23, 1983.

* Department of Mathematics, Hiroshima University, Hiroshima 730, Japan. 
In the case of competition-diffusion systems involving two unknowns, as far as those with spatially-homogeneous coefficients and with homogeneous Neumann boundary conditions are concerned (see (1a)-(1b) below), no rigorous study has ever succeeded in proving the existence of a stable spatially-in homogeneous equilibrium solution. This is in marked contrast to the case of three or more unknowns, where one can easily show the existence of such an equilibrium (or periodic) solution through the usual bifurcation method (see Kishimoto [9], Kishimoto, Mimura and Yoshida [10]). The difficulty in the present problem mainly lies in the fact that, in the case of two unknowns - unlike the case of three or more-, any spatially-inhomogeneous equilibrium solution bifurcating from a spatially-homogeneous one (simple primary bifurcation) is unstable, hence one needs to trace the bifurcation branch at least up to the secondary bifurcation point (which of course is hard to do) if he ever tries to find a stable spatially-inhomogeneous equilibrium solution through the bifurcation analysis. Hale and Vegas ([5], [24]; see also Keyfitz and Kuiper [7]) have succeeded in this approach in the case of single equations in their attempt to give a bifurcation theoretical version of the results by Matano [14], one of the present authors, on pattern formation in nonconvex domains. The bifurcation equations for competition-diffusion systems, however, are much more complicated than those for single equations and, therefore, seem to be all the more difficult to deal with.

In this paper we first consider the following system of equations with unknowns $u=u(x, t), v=v(x, t)$ :

$$
\begin{cases}\frac{\partial u}{\partial t}=d_{1} \Delta u+f(u, v) & (x \in \Omega, t>0) \\ \frac{\partial v}{\partial t}=d_{2} \Delta v+g(u, v) & (x \in \Omega, t>0)\end{cases}
$$

where $d_{1}, d_{2}$ are positive constants, $f, g$ are smooth mappings of $\boldsymbol{R}^{2}$; into $\boldsymbol{R}$ and $\Omega$ is a bounded domain in $\boldsymbol{R}^{n}$; with smooth boundary $\partial \Omega$. We impose homogeneous Neumann boundary conditions

$$
\frac{\partial u}{\partial n}=\frac{\partial v}{\partial n}=0 \quad(x \in \partial \Omega, t>0),
$$

where $\partial / \partial n$ denotes the outer normal derivative on $\partial \Omega$, and the initial conditions

$$
u(x, 0)=u_{0}(x), \quad v(x, 0)=v_{0}(x) \quad(x \in \Omega),
$$

where $u_{0}, v_{0}$ are continuous functions on $\bar{\Omega}$. 
The system (1a)-(1c), which we hereafter simply denote by (1), is said to be of competition type if the inequalities

$$
\frac{\partial f}{\partial v}(u, v) \leqq 0, \quad \frac{\partial g}{\partial u}(u, v) \leqq 0
$$

hold for any $u$ and $v$. In this paper we shall always assume (2) and call the system (1) a competition-diffusion system. The system (1) appears as a simple but important mathematical model in many fields of sciences such as population dynamics in mathematical ecology, population genetics, morphogenesis, chemical reactor theory, etc.; and the physical interpretation of condition (2) is that the two species $u, v$ are competing with each other so that the increase of the population of one species reduces the growth rate of the other.

A pair $(u, v)=(u(x), v(x))$ is called an equilibrium solution of $(1)$ if it is a solution of the elliptic boundary value problem

$$
\begin{cases}d_{1} \Delta u+f(u, v)=0 & (x \in \Omega) \\ d_{2} \Delta v+g(u, v)=0 & (x \in \Omega) \\ \frac{\partial u}{\partial n}=\frac{\partial v}{\partial n}=0 & (x \in \partial \Omega) .\end{cases}
$$

Kishimoto [9] proved that any nonconstant (i.e. spatially-inhomogeneous) equilibrium solution of the competition-diffusion system (1) - with any $d_{1}, d_{2}$ $>0$ and any $f, g$ satisfying (2) - is unstable if $\Omega$ is a rectangular parallelepiped region in $\boldsymbol{R}^{n}$; this result has recently been extended by Kishimoto and Weinberger [11] to the case where $\Omega$ is any bounded convex domain. Their instability result is a generalized version of those of Casten and Holland [2] and Matano [14] (which are on single equations), and, when coupled with the instability results on periodic solutions (Hirsch [6]; see also Theorem 2.4 of the present paper), implies that the phenomenon of pattern formation never occurs if $\Omega$ is bounded and convex; more precisely, almost all the bounded solutions converge to some constant (i.e., spatially-homogeneous) equilibrium solutions as $t \rightarrow+\infty$.

On the other hand, when $\Omega$ is not necessarily convex, not much has been known about the possibility of occurrence of pattern formation. One of the few noteworthy known results is that all solutions of (1) tend to spatially-homogeneous ones at $t \rightarrow+\infty$ if the diffusion coefficients $d_{1}, d_{2}$ are larger than a certain constant that depends on $f, g$ and $\Omega$; hence no occurrence of pattern formation in this case. (See, for instance, Conway, Hoff and Smoller [3]; see also Brown, 
[1] for another kind of instability - or rather nonexistence - result.) Yet the question still remains to be answered as to whether there exists, after all, a stable spatially-inhomogeneous equilibrium solution of (1) if $d_{1}, d_{2}, f, g, \Omega$ are taken appropriately; the results [11], [3] mentioned above only suggest that $\Omega$ cannot be convex and that $d_{1}, d_{2}$ cannot be so large if (1) ever possesses such an equilibrium solution. One of the main purposes of the present paper is to give an affirmative answer to this question (Theorem $\mathrm{A}$ in Section 3).

In Section 4, we consider a system of quasilinear diffusion equations of the form

$$
\begin{cases}\frac{\partial u}{\partial t}=\Delta\left\{\left(d_{1}+\alpha v\right) u\right\}+f(u, v) & (x \in \Omega, t>0) \\ \frac{\partial v}{\partial t}=\Delta\left\{\left(d_{2}+\beta u\right) v\right\}+g(u, v) & (x \in \Omega, t>0)\end{cases}
$$

under the homogeneous Neumann boundary conditions, where $d_{1}, d_{2}, f, g, \Omega$ are as in (1) and $\alpha, \beta$ are some nonnegative constants. This system arises as a mathematical model describing the population distribution of two species which are interacting with each other in a certain manner while "biodiffusing" over the domain $\Omega$ (Shigesada et al [23], Mimura [16]). The terms $d_{1}+\alpha v, d_{2}+\beta u$ represent "biodiffusion" coefficients; in the present case the biodiffusioncoefficient of one species depends on the population density of the other species, and we call $\alpha, \beta$ cross-population pressures. The meaning of "biodiffusion" will be made clear in Section 4.

In the case $\alpha=\beta=0$, (4) reduces to the semilinear system (1); and it is already mentioned above that $\Omega$ needs to be convex in this case for the occurrence of pattern formation. However, in the case where $\alpha>0$ and/or $\beta>0$, the system (4) exhibits a dynamical behavior that is quite different from that of (1). And we are particularly interested to see how the presence of cross-population pressures contributes to the occurrence of pattern formation, which, in fact, often occurs in this case even when $\Omega$ is a convex domain.

\section{§ 2. Strongly Order-Preserving Local Semiflows}

One of the notable characteristics of competition-diffusion systems for two species is the so-called comparison principle, which is derived from the maximum principle. Owing to this property, the general theory of strongly order-preserving local semiflows recently established by Hirsch [6] and Matano [15] 
applies to the present system (1), thereby yielding a number of preliminary theorems on the dynamical structure of this system. (Note that the comparison principle does not hold for competition systems with three or more unknowns or with cross-diffusion terms.)

The comparison principle is stated as follows: Let $(u, v),(\bar{u}, \bar{v})$ be solutions to (1) with initial data $\left(u_{0}, v_{0}\right),\left(\bar{u}_{0}, \bar{v}_{0}\right)$ respectively. Suppose

$$
\bar{u}_{0}(x) \geqq u_{0}(x), \quad \bar{v}_{0}(x) \leqq v_{0}(x)
$$

hold for all $x \in \bar{\Omega}$. Then

$$
\bar{u}(x, t) \geqq u(x, t), \quad \bar{v}(x, t) \leqq v(x, t)
$$

for all $x \in \bar{\Omega}$ and any $t \geqq 0$.

The above property of the system (1) suggests us to introduce an order relation into the space $C(\bar{\Omega}) \times C(\bar{\Omega})$ in the following manner:

$$
\left(\begin{array}{l}
\bar{\phi} \\
\bar{\psi}
\end{array}\right) \geqq\left(\begin{array}{l}
\phi \\
\psi
\end{array}\right) \Longleftrightarrow \begin{aligned}
& \bar{\phi}(x) \geqq \phi(x) \\
& \bar{\psi}(x) \leqq \psi(x)
\end{aligned} \text { in } \bar{\Omega} .
$$

In what follows we regard $C(\bar{\Omega}) \times C(\bar{\Omega})$ as an ordered Banach space with respect to the above relation. It is clear that the system (1) defines a local semiflow on $C(\bar{\Omega}) \times C(\bar{\Omega})$ and that this local semiflow preserves the order relation just defined above. Actually, as will be seen in Proposition 2.1 below, a stronger version of the comparison principle holds for this local semiflow.

Definition 1. Let $X$ be an open subset of an ordered Banach space with order relation $\geqq$ and let $\Phi=\Phi(t), t \geqq 0$, be a local semiflow on $X$. We say $\Phi$ is strongly order-preserving if, for any pair of points $p, q$ in $X$ with $p \geqq q, p \neq q$ and for any $t>0$ (for which both $\Phi(t) p, \Phi(t) q$ are definable), there exist open sets $V \ni p, W \ni q$ such that

$$
\Phi(t) p^{\prime} \geqq \Phi(t) q^{\prime}
$$

holds for any $p^{\prime} \in V, q^{\prime} \in W$.

Proposition 2.1. Let $\Phi$ be the local semiflow defined by (1). Assume

$$
\frac{\partial f}{\partial v}<0, \quad \frac{\partial g}{\partial u}<0
$$

for all $u, v$. Then $\Phi$ is strongly order-preserving with respect to the order relation (5).

Remark 2.2. If we simply assume (2) instead of (6), then $\Phi$ is order-pre- 
serving but not necessarily strongly order-preserving. However, it still has the following property: Let $p, q \in C(\bar{\Omega}) \times C(\bar{\Omega})$ satisfy $p \gg q$, then there exist open sets $V, W$ satisfying the conditions in Definition 1 . Here we have used the notation

$$
\left(\begin{array}{l}
\bar{\phi} \\
\bar{\psi}
\end{array}\right) \gg\left(\begin{array}{l}
\phi \\
\psi
\end{array}\right) \Longleftrightarrow \begin{aligned}
& \bar{\phi}(x) \geqq \phi(x), \bar{\phi}(x) \not \equiv \phi(x) \\
& \bar{\psi}(x) \leqq \psi(x), \bar{\psi}(x) \not \equiv \psi(x) .
\end{aligned}
$$

This property of $\Phi$ stems from the strong maximum principle. If we assume, in addition to (2), that

(8) neither $\left\{(u, v) \mid f_{v}(u, v)=0\right\}$ nor $\left\{(u, v) \mid g_{u}(u, v)=0\right\}$ has interior points,

(9a) $\{(u, v) \mid f(u, v)=0\}$ does not contain a line segment parallel to the $v$-axis,

(9b) $\{(u, v) \mid g(u, v)=0\}$ does not contain a line segment parallel to the $u$-axis, then $\Phi$ is strongly order-preserving.

Another important property of $\Phi$ is its compactness; that is, for any bounded set $B$ in $C(\bar{\Omega}) \times C(\bar{\Omega})$, there exists a positive number $t_{0}$ such that $\Phi(t) B$ is relatively compact for each $t, 0<t<t_{0}$. By virtue of these properties of $\Phi$, we can apply the general theory of [6], [15], to get the following theorems on the dynamical structure of (1):

Theorem 2.3 (Hirsch). Assume (2), (8), (9) hold. Then "almost" all bounded solutions of (1) are quasi-convergent; in other words, their $\omega$-limit sets consist only of equilibrium solutions.

A careful discussion shows that we can drop the assumptions (8), (9) in Theorem 2.2. As an immediate corollary to this theorem, we have

Theorem 2.4 (Hirsch). Under the assumption (2), any periodic solution of (1), if existing, is unstable.

A pair of functions $(u, v)=(u(x), v(x))$ is called an equilibrium solution of (1) if it satisfies (3). Note that $p \in C(\bar{\Omega}) \times C(\bar{\Omega})$ is an equilibrium solution if and only if

$$
\Phi(t) p=p
$$

for all $t \geqq 0$.

Definition 2. $q \in C(\bar{\Omega}) \times C(\bar{\Omega})$ is called a (time-independent) supersolution (resp. subsolution) on (1) if 


\section{$\Phi(t) q \leqq q \quad($ resp. $\Phi(t) q \geqq q)$}

for any $t \geqq 0$. If, in addition, $q$ is not an equilibrium solution, then it is called a strict supersolution (resp. strict subsolution).

Definition 3. Let $p \in C(\bar{\Omega}) \times C(\bar{\Omega})$ be an equilibrium solution of (1). We say $p$ is stable if for any neighborhood $V$ of $p$ there exists a neighborhood $W$ of $p$ such that $\Phi(t) W \subset V$ for all $t \geqq 0$. We say $p$ is unstable if it is not stable.

Theorem 2.5 (Matano). Assume (2) holds. Then any unstable equilibrium solution $p$ of (1) has a nontrivial unstable set; that is, there exists a negative half orbit (other than $\{p\}$ itself) that converges to $p$ as $t \rightarrow-\infty$.

Theorem 2.6 (Matano). Assume (2), (9) hold. Let $\bar{q}, q \in C(\bar{\Omega}) \times C(\bar{\Omega})$ be a strict supersolution and a strict subsolution of (1) respectively such that $\bar{q} \geqq q, \bar{q} \neq q$. Then there exists a stable equilibrium solution $p$ of (1) satisfying $\bar{q} \geqq p \geqq q$.

\section{§3. Pattern Formation in Nonconvex Domains}

The following is one of the main theorems of this paper:

Theorem A. Assume that (2) and (8) (or (2) and (9)) hold and that the system of ordinary differential equations

$$
\left\{\begin{array}{l}
\frac{d u}{d t}=f(u, v) \\
\frac{d v}{d t}=g(u, v)
\end{array}\right.
$$

possesses a pair of asymptotically stable equilibria $p_{1}, p_{2}$ with $p_{1} \gg p_{2}$, where $\gg$ is as in (7). Assume also that $n \geqq 2$. Then, for any $d_{1}>0, d_{2}>0$, we can find a bounded domain $\Omega$ in $\boldsymbol{R}^{n}$ for which (1) possesses a stable spatiallyinhomogeneous equilibrium solution.

Remark 3.1. The condition $p_{1} \gg p_{2}$ implies that $p_{1}=\left(\bar{u}_{1}, \bar{v}_{1}\right), p_{2}=\left(\bar{u}_{2}, \bar{v}_{2}\right)$ satisfy $\bar{u}_{1}>\bar{u}_{2}, \bar{v}_{1}<\bar{v}_{2}$. Note that the assumptions (2), (8), (9) are needed only in the rectangular region $\left\{(u, v) \in \boldsymbol{R}^{2} \mid \bar{u}_{1}>u>\bar{u}_{2}, \bar{v}_{1}<v<\bar{v}_{2}\right\}$.

Lemma 3.2. Suppose $q=(u, v)$ satisfies $u, v \in C^{1}(\bar{\Omega}) \cap C^{2}(\Omega)$ and that 


$$
\begin{cases}d_{1} \Delta u+f(u, v) \leqq 0 & \text { in } \quad \Omega \\ d_{2} \Delta v+g(u, v) \geqq 0 & \text { in } \quad \Omega \\ \frac{\partial u}{\partial n} \geqq 0, \quad \frac{\partial v}{\partial n} \leqq 0 & \text { on } \quad \partial \Omega .\end{cases}
$$

Then $q=(u, v)$ is a supersolution of (1). If $q$ satisfies the reversed differential inequalities, then it is a subsolution of (1).

The above lemma follows immediately from the maximum principle.

Remark 3.3. Lemma 3.2 can be extended to the case where $q$ is piecewise smooth: Let $S$ be a smooth hypersurface dividing $\Omega$ into disjoint regions $\Omega_{1}$, $\Omega_{2}$; namely, $\Omega=\Omega_{1} \cup S \cup \Omega_{2}$. Suppose $q=(u, v) \in C(\bar{\Omega}) \times C(\bar{\Omega})$ is $C^{1}$ on each $\bar{\Omega}_{1}, \bar{\Omega}_{2}$ and $C^{2}$ on each $\Omega_{1}, \Omega_{2}$. Suppose further that (11) holds except on $S$ and that

$$
\frac{\partial u}{\partial \xi}+\frac{\partial u}{\partial \zeta} \leqq 0, \frac{\partial v}{\partial \xi}+\frac{\partial v}{\partial \zeta} \geqq 0 \quad \text { on } \quad S,
$$

where $\partial / \partial \xi$, $\partial / \partial \zeta$ denote the inner normal derivatives on $\partial \Omega_{1} \cap S, \partial \Omega_{2} \cap S$ respectively. Then $q$ is a supersolution of (1).

In order to prove this assertion, use Green's formula to see that (11) holds on $\bar{\Omega}$ in a generalized sense; then apply Sattinger's result [20; Theorem 3.4].

Lemma 3.4. Assume that (2) holds and that (5) possesses a pair of asymptotically stable equilibria $p_{1}, p_{2}$ with $p_{1} \gg p_{2}$, where $\gg$ is as in Remark 3.1. Then there exist positive numbers $\delta, h$ and square regions

$$
\begin{array}{ll}
R_{1}=\left\{(u, v) \in \boldsymbol{R}^{2} \mid\right. & \left.\left|u-a_{1}\right| \leqq h,\left|v-b_{1}\right| \leqq h\right\}, \\
R_{2}=\left\{(u, v) \in \boldsymbol{R}^{2} \mid\right. & \left.\left|u-a_{2}\right| \leqq h,\left|v-b_{2}\right| \leqq h\right\},
\end{array}
$$

with $p_{1} \gg R_{1} \gg R_{2} \gg p_{2}$ such that

$$
\begin{array}{lll}
f(u, v) \geqq \delta, \quad g(u, v) \leqq-\delta & \text { in } & R_{1}, \\
f(u, v) \leqq-\delta, g(u, v) \geqq \delta & \text { in } & R_{2} .
\end{array}
$$

Proof. Using the order-preserving property of (10), one can easily show the existence of solutions $\left(u_{1}(t), v_{1}(t)\right),\left(u_{2}(t), v_{2}(t)\right)$ to $(10)$ such that

$$
\begin{array}{ll}
\left(u_{1}(t), v_{1}(t)\right) \longrightarrow p_{1} \quad \text { increasingly as } & t \longrightarrow \infty, \\
\left(u_{2}(t), v_{2}(t)\right) \longrightarrow p_{2} \quad \text { decreasingly as } t \longrightarrow \infty,
\end{array}
$$

where the monotonicity of these solutions is with respect to the relation $\gg$. (See 
[15; Lemma 3.2 and Lemma 5.11]. Although these lemmas are on strongly order-preserving local semiflows, the same argument applies to the system (10) if we simply assume (2).) The conclusion of Lemma 3.4 now follows immediately.

Next let us specify the shape of the domain $\Omega$. In what follows we shall mainly consider the case $n=2$, since the case $n \geqq 3$ can be treated quite similarly.

Let $\Omega_{R}, \Omega_{L}$ be bounded domains in $R^{2}$ with smooth boundaries $\partial \Omega_{R}, \partial \Omega_{L}$ such that

$$
\begin{aligned}
& \Omega_{R} \subset\left\{\left(x_{1}, x_{2}\right) \in \boldsymbol{R}^{2} \mid x_{1}>l\right\}, \\
& \Omega_{L} \subset\left\{\left(x_{1}, x_{2}\right) \in \boldsymbol{R}^{2} \mid x_{1}<-l\right\}
\end{aligned}
$$

for some $l>0$ and that

$$
\begin{aligned}
& \partial \Omega_{R} \cap\left\{\left(x_{1}, x_{2}\right) \in \boldsymbol{R}^{2} \mid x_{1}=l\right\} \supset\left\{\left(l, x_{2}\right)|\quad| x_{2} \mid \leqq m\right\}, \\
& \partial \Omega_{L} \cap\left\{\left(x_{1}, x_{2}\right) \in \boldsymbol{R}^{2} \mid x_{1}=-l\right\} \supset\left\{\left(-l, x_{2}\right)|\quad| x_{2} \mid \leqq m\right\}
\end{aligned}
$$

for some $m>0$. Let $r=r(s),-l \leqq s \leqq l$, be a smooth function satisfying the following conditions:

$$
\begin{aligned}
& r(s)>0 \quad(-l \leqq s \leqq l), \\
& \max _{|s| \leqq l} r(s) \equiv r_{M} \leqq m, \\
& \lim _{s \uparrow l} r^{\prime}(s)=+\infty, \quad \lim _{s \downarrow-l} r^{\prime}(s)=-\infty, \\
& \lim _{s \rightarrow \pm l} r^{\prime \prime}(s) /\left\{r^{\prime}(s)\right\}^{3}=0, \quad r^{\prime \prime}(0)=0 .
\end{aligned}
$$

Set

$$
r_{\varepsilon}(s)= \begin{cases}\varepsilon r\left(\frac{s-l+l \varepsilon}{\varepsilon}\right) & (l-l \varepsilon \leqq s \leqq l) \\ \varepsilon r(0) & (-l+l \varepsilon \leqq s \leqq l-l \varepsilon) \\ \varepsilon r\left(\frac{s+l-l \varepsilon}{\varepsilon}\right) & (-l \leqq s \leqq-l+l \varepsilon) .\end{cases}
$$

And put

$$
\begin{aligned}
& D_{\varepsilon}=\left\{\left(x_{1}, x_{2}\right)|| x_{2} \mid<r_{\varepsilon}(s),-l \leqq x_{1} \leqq l\right\}, \\
& \Omega_{\varepsilon}=\Omega_{L} \cup D_{\varepsilon} \cup \Omega_{R}
\end{aligned}
$$

for $0<\varepsilon \leqq 1$ (see Fig. 1$)$. The conditions $(13 \mathrm{c}),(13 \mathrm{~d})$ imply that $\Omega_{\varepsilon}$ has smooth boundary of class $C^{2}$. 


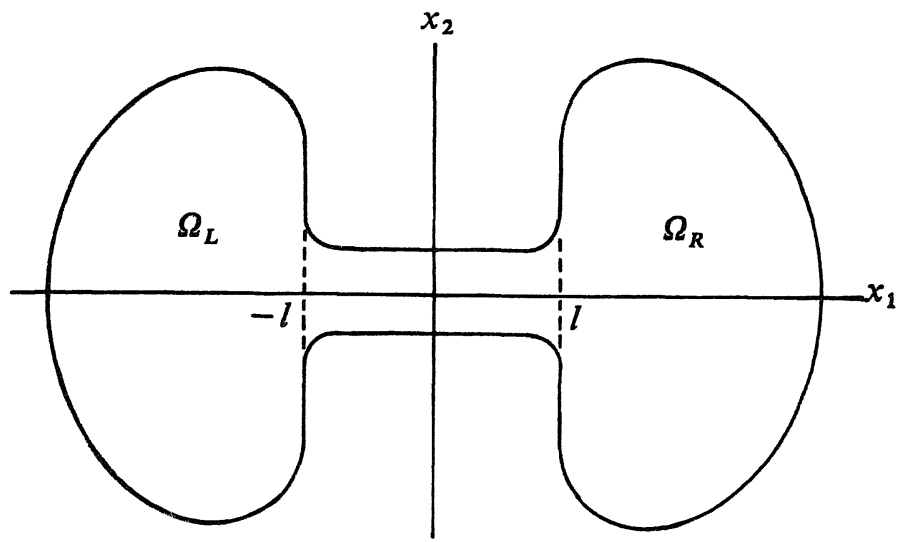

Fig. 1.

Now let $\lambda_{\varepsilon}, \mu_{\varepsilon}$ be the least eigenvalues of the problems

$$
\begin{aligned}
& \begin{cases}\Delta \varphi+\lambda \varphi=0 & \text { in } G_{\varepsilon}^{+} \\
\frac{\partial \varphi}{\partial n}=0 & \text { on } \Gamma_{\varepsilon}^{+} \\
\varphi=0 & \text { on } \gamma_{\varepsilon}^{+},\end{cases} \\
& \begin{cases}\Delta \psi+\mu \psi=0 & \text { in } G_{\varepsilon}^{-} \\
\frac{\partial \psi}{\partial n}=0 & \text { on } \Gamma_{\varepsilon}^{-} \\
\psi=0 & \text { on } \gamma_{\varepsilon}^{-},\end{cases}
\end{aligned}
$$

respectively, where

$$
\begin{aligned}
& G_{\varepsilon}^{ \pm}=\Omega_{\varepsilon} \cap\left\{\left(x_{1}, x_{2}\right) \in \boldsymbol{R}^{2} \mid \pm x_{1}>l-l \varepsilon\right\}, \\
& \Gamma_{\varepsilon}^{ \pm}=\partial \Omega_{\varepsilon} \cap\left\{\left(x_{1}, x_{2}\right) \in \boldsymbol{R}^{2} \mid \pm x_{1}>l-l \varepsilon\right\}, \\
& \gamma_{\varepsilon}^{ \pm}=\Omega_{\varepsilon} \cap\left\{\left(x_{1}, x_{2}\right) \in \boldsymbol{R}^{2} \mid \pm x_{1}=l-l \varepsilon\right\} .
\end{aligned}
$$

By the Krein-Rutman theory, both $\lambda_{\varepsilon}, \mu_{\varepsilon}$ are simple for each $0<\varepsilon \leqq 1$, and the corresponding eigenfunctions do not change sign in $G_{\varepsilon}^{+}, G_{\varepsilon}^{-}$respectively.

Lemma 3.5. Let $\left|\Omega_{R}\right|,\left|\Omega_{J}\right|$ denote the area of domains $\Omega_{R}, \Omega_{L}$ respectively. Then

$$
\lambda_{\varepsilon}<\frac{2 r_{M}}{l\left|\Omega_{R}\right|}, \quad \mu_{\varepsilon}<\frac{2 r_{M}}{l\left|\Omega_{L}\right|}
$$

for all $\varepsilon, 0<\varepsilon \leqq 1$, where $r_{M}$ is as in (13b). 
Proof. Given a domain $\Omega \subset R^{2}$ and a positive integer $j$, denote by $H^{j}(\Omega)$ the space of all $L^{2}(\Omega)$ functions whose derivatives of order up to $j$ all belong to $L^{2}(\Omega)$. It is easily seen that

$$
\lambda_{\varepsilon}=\min _{w \in A_{\varepsilon}} \int_{G_{\varepsilon}^{+}}|\nabla w|^{2} d x / \int_{G_{\varepsilon}^{+}} w^{2} d x,
$$

where

$$
A_{\varepsilon}=\left\{w \in H^{1}\left(G_{\varepsilon}^{+}\right) \mid \quad w=0 \quad \text { on } \quad \gamma_{\varepsilon}^{+}, \quad w \not \equiv\right\} .
$$

Now define a function $w_{0}$ on $\overline{G_{\varepsilon}^{+}}$by

$$
w_{0}\left(x_{1}, x_{2}\right)= \begin{cases}1 & \text { if } x_{1}>l \\ \frac{x_{1}-l+l \varepsilon}{l \varepsilon} & \text { if } x_{1} \leqq l .\end{cases}
$$

Obviously $w_{0}$ belongs $A_{\varepsilon}$ and

$$
\frac{\int_{G_{\varepsilon}^{+}}\left|\nabla w_{0}\right|^{2} d x}{\int_{G_{t}^{+}}\left(w_{0}\right)^{2} d x}<\frac{2 r_{M}}{l\left|\Omega_{R}\right|} .
$$

Combining this and (18), we get the first inequality in Lemma 3.5. The second inequality can be shown likewise.

As a matter of fact, it can further be shown that $\lambda_{\varepsilon} \rightarrow 0, \mu_{\varepsilon} \rightarrow 0$ as $\varepsilon \rightarrow 0$, but we shall not need this in the later discussion.

Let us now consider the boundary value problems

$$
\begin{aligned}
& \begin{cases}\Delta w+\sigma_{1} w\left(1-w^{2}\right)=0 & \left(x \in G_{\varepsilon}^{+}\right) \\
\frac{\partial w}{\partial n}=0 & \left(x \in \Gamma_{\varepsilon}^{+}\right) \\
w=0 & \left(x \in \gamma_{\varepsilon}^{+}\right),\end{cases} \\
& \begin{cases}\Delta W+\sigma_{2} W\left(1-W^{2}\right)=0 & \left(x \in G_{\varepsilon}^{-}\right) \\
\frac{\partial W}{\partial n}=0 & \left(x \in \Gamma_{\varepsilon}^{-}\right) \\
W=0 & \left(x \in \gamma_{\varepsilon}^{-}\right),\end{cases}
\end{aligned}
$$

where

$$
\sigma_{1}=\frac{4 r_{M}}{l\left|\Omega_{R}\right|}, \quad \sigma_{2}=\frac{4 r_{M}}{l\left|\Omega_{L}\right|} .
$$


Using Lemma 3.5 and the fact that the function $w\left(1-w^{2}\right)$ is concave in $w \geqq 0$, one easily finds that (20), (21) each possess a unique positive solution, which we shall denote by $w_{\varepsilon}, W_{\varepsilon}$ respectively.

\section{Lemma 3.6.}

$$
\begin{array}{ll}
0<W_{\varepsilon}(x)<1 & \left(x \in G_{\varepsilon}^{+}\right), \\
0<W_{\varepsilon}(x)<1 & \left(x \in G_{\varepsilon}^{-}\right)
\end{array}
$$

for all $\varepsilon, 0<\varepsilon \leqq 1$.

The above lemma follows immediately from the maximum principle, so we omit the proof.

\section{Lemma 3.7.}

$$
\begin{aligned}
& \int_{G_{\varepsilon}^{+}} w_{\varepsilon}^{2} d x \geqq \frac{1}{8}\left|\Omega_{R}\right|, \\
& \int_{G_{\varepsilon}^{-}} W_{\varepsilon}^{2} d x \geqq \frac{1}{8}\left|\Omega_{L}\right|
\end{aligned}
$$

for all $\varepsilon, 0<\varepsilon \leqq 1$.

Proof. We only prove the former inequality, since the latter can be shown quite similarly. Consider the functional

$$
J(w)=\frac{1}{2} \int_{G_{\varepsilon}^{+}}\left\{|\nabla w|^{2}-\sigma_{1} w^{2}\left(1-\frac{w^{2}}{2}\right)\right\} d x
$$

associated with the variational formulation of the problem (20). Since $w_{\varepsilon}$ is the only stable solution to (20), we easily find that

$$
J\left(w_{\varepsilon}\right)=\min _{w \in X_{\varepsilon}} J(w),
$$

where $X_{\varepsilon}=\left\{w \in H^{1}\left(G_{\varepsilon}^{+}\right) \mid w=0\right.$ on $\left.\gamma_{\varepsilon}^{+}\right\}$. Let $w_{0}$ be as in (19). A simple calculation yields

$$
J\left(\frac{1}{\sqrt{2}} w_{0}\right) \leqq-\frac{\sigma_{1}}{16}\left|\Omega_{R}\right|
$$

Consequently,

$$
-\frac{\sigma_{1}}{16}\left|\Omega_{R}\right| \geqq J\left(w_{\varepsilon}\right) \geqq-\frac{\sigma_{1}}{2} \int_{G_{\varepsilon}^{+}} w_{\varepsilon}^{2}\left(1-\frac{w_{\varepsilon}^{2}}{2}\right) d x,
$$

from which follows the conclusion of Lemma 3.7. 


\section{Lemma 3.8.}

$$
\begin{aligned}
& \int_{\gamma_{\varepsilon}^{+}} \frac{\partial w_{\varepsilon}}{\partial n} d x_{2}=\sigma_{1} \int_{G_{\varepsilon}^{+}} w_{\varepsilon}\left(1-w_{\varepsilon}^{2}\right) d x, \\
& \int_{G_{\varepsilon}^{+}}\left|\nabla w_{\varepsilon}\right|^{2} d x=\sigma_{1} \int_{G_{\varepsilon}^{+}} w_{\varepsilon}^{2}\left(1-w_{\varepsilon}^{2}\right) d x .
\end{aligned}
$$

Proof. Obvious by Green's formula.

Lemma 3.9. There exist positive constants $K_{1}, K_{2}$ such that

$$
\begin{aligned}
& \int_{G_{\varepsilon}^{+}} w_{\varepsilon}^{2}\left(1-{w_{\varepsilon}}^{2}\right) d x \geqq \frac{K_{1}}{|\log \varepsilon|+1}, \\
& \int_{G_{\varepsilon}^{-}} W_{\varepsilon}^{2}\left(1-W_{\varepsilon}^{2}\right) d x \geqq \frac{K_{2}}{|\log \varepsilon|+1}
\end{aligned}
$$

for all $\varepsilon, 0<\varepsilon \leqq 1$.

Proof. We only prove the former inequality since the latter can be shown quite similarly.

For each $s, 0<s \leqq 1$, let $C_{s}$ be a circle of radius $r_{M} s$ centered at the point $(l, 0)$ and set

$$
\Gamma_{s}=\Omega_{R} \cap C_{s}
$$

for $0<s \leqq 1$. Denote by $A_{\varepsilon, s}$ the set of points in $G_{\varepsilon}^{+}$lying on the left of $\Gamma_{s}$. Also, set

$$
\begin{aligned}
& a(\varepsilon)=\int_{G_{\varepsilon}^{+}} w_{\varepsilon}^{2}\left(1-w_{\varepsilon}^{2}\right) d x, \\
& I_{\varepsilon}(s)=\int_{\Gamma_{s}} w_{\varepsilon}^{2} d \bar{x} .
\end{aligned}
$$

By (20) and Green's formula, it holds that

$$
\begin{aligned}
\int_{\Gamma_{s}} w_{\varepsilon} \frac{\partial w_{\varepsilon}}{\partial \xi} d \bar{x} & =\int_{G_{\varepsilon}^{+} \backslash A_{\varepsilon}, s}\left\{\sigma_{1} w_{\varepsilon}{ }^{2}\left(1-w_{\varepsilon}{ }^{2}\right)-\left|\nabla w_{\varepsilon}\right|^{2}\right\} d x \\
& \leqq \sigma_{1} a(\varepsilon),
\end{aligned}
$$

where $\partial / \partial \xi$ denotes the outer normal derivative to $\Gamma_{s} \subset \partial A_{\varepsilon, s^{*}}$ Consequently, we have

$$
\begin{aligned}
\frac{d}{d s} I_{\varepsilon}(s) & =\frac{1}{s} I_{\varepsilon}(s)+2 \int_{\Gamma_{s}} \frac{\partial w_{\varepsilon}}{\partial \xi} W_{\varepsilon} d \bar{x} \\
& \leqq \frac{1}{s} I_{\varepsilon}(s)+2 \sigma_{1} a(\varepsilon),
\end{aligned}
$$


from which it follows that

$$
I_{\varepsilon}(s) \leqq \frac{s}{\varepsilon} I_{\varepsilon}(\varepsilon)+2 \sigma_{1} a(\varepsilon) s \log \frac{s}{\varepsilon}
$$

for $s \in[\varepsilon, 1]$. The boundary condition $w_{\varepsilon}=0\left(\right.$ on $\gamma_{\varepsilon}^{+}$) yields the estimate

$$
\begin{aligned}
I_{\varepsilon}(\varepsilon) & =\int_{\Gamma_{\varepsilon}} w_{\varepsilon}{ }^{2} d \bar{x} \leqq C \varepsilon \int_{A_{\varepsilon, \varepsilon}}\left|\nabla w_{\varepsilon}\right|^{2} d x \\
& \leqq C \varepsilon \int_{G_{\varepsilon}^{+}}\left|\nabla w_{\varepsilon}\right|^{2} d x=C \sigma_{1} a(\varepsilon),
\end{aligned}
$$

where $C$ is a positive constant independent of $\varepsilon$. Combining this and (25), we see that

$$
I_{\varepsilon}(s) \leqq \sigma_{1} a(\varepsilon)\left(C+2 \log \frac{s}{\varepsilon}\right) s .
$$

Also, a similar calculation shows that there exists a positive constant, denoted by $\bar{C}$, such that

$$
\begin{aligned}
\int_{A_{\varepsilon, 1}} w_{\varepsilon}^{2} d x & =\int_{A_{\varepsilon, \varepsilon}} w_{\varepsilon}^{2} d x+\int_{A_{\varepsilon, 1} \mid A_{\varepsilon, \varepsilon}} w_{\varepsilon}^{2} d x \\
& \leqq \bar{C} \sigma_{1} \varepsilon^{2} a(\varepsilon)+\int_{\varepsilon}^{1} I_{\varepsilon}(s) d s
\end{aligned}
$$

hence, by (26),

$$
\int_{A_{\varepsilon, 1}} w_{\varepsilon}^{2} d x<\sigma_{1} a(\varepsilon)\left(M_{1}+|\log \varepsilon|\right),
$$

where $M_{1}$ is a positive constant independent of $\varepsilon$.

Now suppose that the conclusion of Lemma 3.9 (the former inequality) does not hold. Then there exists a sequence $\varepsilon_{1}, \varepsilon_{2}, \ldots$ such that

$$
\lim _{k \rightarrow \infty}\left(1+\left|\log \varepsilon_{k}\right|\right) a\left(\varepsilon_{k}\right)=0 .
$$

Denote by $G$ the set of all points in $\Omega_{R}$ lying on the left of the arc $\Gamma_{1}$; in other words,

$$
G=\Omega_{R} \cap A_{\varepsilon, 1} \text {. }
$$

By (27) and (28) we have

$$
\int_{G} w_{\varepsilon_{k}}^{2} d x \longrightarrow 0 \quad(\text { as } k \longrightarrow \infty) \text {. }
$$

On the other hand, as Lemma 3.6 and (23) imply that $\left\{\left.w_{\varepsilon_{k}}\right|_{\Omega_{R}}\right\}$ is bounded in $H^{1}\left(\Omega_{R}\right)$ - hence relatively compact in $L^{2}\left(\Omega_{R}\right)$-, we may assume without 
loss of generality that

$$
\left.w_{\varepsilon_{k}}\right|_{\Omega_{R}} \longrightarrow \tilde{w} \text { strongly in } L^{2}\left(\Omega_{R}\right) \text { as } k \longrightarrow \infty
$$

for some $\tilde{w} \in L^{2}\left(\Omega_{R}\right)$. The estimate

$$
\int_{\Omega_{R}}\left|\nabla w_{\varepsilon_{k}}\right|^{2} d x<\sigma_{1} a\left(\varepsilon_{k}\right) \longrightarrow 0 \quad(\text { as } k \rightarrow \infty)
$$

implies that $\tilde{w}$ is a constant function, hence, by (29), we have $\tilde{w} \equiv 0$, from which it follows that

$$
\int_{\Omega_{R}} w_{\varepsilon_{k}}^{2} d x \longrightarrow 0 \quad(\text { as } k \rightarrow 0)
$$

Combining this and the estimate

$$
\int_{G_{\varepsilon}^{+} \backslash \Omega_{R}} w_{\varepsilon}{ }^{2} d x<\bar{C} \sigma_{1} \varepsilon^{2} a(\varepsilon)
$$

where $\bar{C}$ is the constant given above, we obtain

$$
\int_{G_{\varepsilon_{k}}^{+}} w_{\varepsilon_{k}}^{2} d x \longrightarrow 0 \quad(\text { as } k \rightarrow \infty)
$$

which contradicts the conclusion of Lemma 3.7. This contradiction shows that the supposition (28) is false, thus completing the proof of Lemma 3.9.

\section{Lemma 3.10.}

$$
\left.\min _{x \in \gamma_{\varepsilon}^{+}} \frac{\partial w_{\varepsilon}}{\partial x_{1}} \longrightarrow+\infty, \max _{x \in \gamma_{\varepsilon}^{-}} \frac{\partial W_{\varepsilon}}{\partial x_{1}} \longrightarrow-\infty \quad \text { (as } \varepsilon \rightarrow 0\right) .
$$

Proof. By (22) and Lemma 3.9, we have

$$
\frac{1}{\varepsilon} \int_{\gamma_{\varepsilon}^{+}} \frac{\partial w_{\varepsilon}}{\partial x_{1}} d x_{2} \longrightarrow+\infty \quad(\text { as } \varepsilon \rightarrow 0)
$$

The former half of Lemma 3.10 follows from this and the strong maximum principle known as the Hopf boundary lemma as well as some compactness argument. Note that we need a generalized version of Hopf lemma due to Serrin [22; Lemma 2] near the end points of $\overline{\gamma_{\varepsilon}^{+}}$. Further details of the proof are omitted.

Proof of Theorem $A$. Let $p_{1}=\left(\bar{u}_{1}, \bar{v}_{1}\right), p_{2}=\left(\bar{u}_{2}, \bar{v}_{2}\right)$ be as in the statement of Theorem A and Lemma 3.4, and let $M>0$ be a constant such that

$$
|f(u, v)| \leqq M, \quad|g(u, v)| \leqq M
$$


hold in the rectangular region

$$
\Sigma=\left\{(u, v) \in \boldsymbol{R}^{2} \mid \bar{u}_{1} \geqq u \geqq \bar{u}_{2}, \bar{v}_{1} \leqq v \leqq \bar{v}_{2}\right\} .
$$

Let $a_{1}, b_{1}, h, \delta$ be as in Lemma 3.4 and define functions $\tilde{u}_{\varepsilon}(x), \tilde{v}_{\varepsilon}(x)$ on $\bar{\Omega}_{\varepsilon}$ by

$$
\begin{aligned}
& \tilde{u}_{\varepsilon}(x)=\left\{\begin{array}{lc}
a_{1}-h+\alpha_{1} w_{\varepsilon}(x) & \text { if } x_{1} \geqq l_{\varepsilon} \\
a_{1}-h+\frac{M}{2 d_{1}}\left(x_{1}-l_{\varepsilon}\right)^{2}+\beta_{1}\left(x_{1}-l_{\varepsilon}\right) & \text { if } x_{1}<l_{\varepsilon},
\end{array}\right. \\
& \tilde{v}_{\varepsilon}(x)= \begin{cases}b_{1}+h-\alpha_{2} w_{\varepsilon}(x) & \text { if } x_{1} \geqq l_{\varepsilon} \\
b_{1}+h-\frac{M}{2 d_{2}}\left(x_{1}-l_{\varepsilon}\right)^{2}-\beta_{2}\left(x_{1}-l_{\varepsilon}\right) & \text { if } x_{1}<l_{\varepsilon},\end{cases}
\end{aligned}
$$

where $\alpha_{i}=\min \left\{2 h, \delta / d_{i} \sigma_{i}\right\}(i=1,2), l_{\varepsilon}=l-l \varepsilon$ and

$$
\begin{aligned}
& \beta_{1}=\frac{M l}{2 d_{1}}+\frac{2\left(a_{1}-h-\bar{u}_{2}\right)}{l}, \\
& \beta_{2}=\frac{M l}{2 d_{2}}+\frac{2\left(\bar{v}_{2}-b_{1}-h\right)}{l} .
\end{aligned}
$$

Finally, set

$$
\begin{aligned}
& \hat{u}_{\varepsilon}(x)= \begin{cases}\max \left\{\tilde{u}_{\varepsilon}(x), \bar{u}_{2}\right\} & \text { (if } \left.x_{1} \geqq 0\right) \\
\bar{u}_{2} & \text { (if } \left.x_{1}<0\right),\end{cases} \\
& \hat{v}_{\varepsilon}(x)= \begin{cases}\min \left\{\tilde{v}_{\varepsilon}(x), \bar{v}_{2}\right\} & \text { (if } \left.x_{1} \geqq 0\right) \\
\bar{v}_{2} & \text { (if } \left.x_{1}<0\right) .\end{cases}
\end{aligned}
$$

From (31) it follows that $\tilde{u}_{\varepsilon}(x) \leqq \bar{u}_{2}, \tilde{v}(x) \geqq \bar{v}_{2}$ (if $x_{1}=0$ ) for any $0<\varepsilon \leqq 1 / 2$, hence $\hat{u}_{\varepsilon}, \hat{v}_{\varepsilon}$ are continuous on $\bar{\Omega}_{\varepsilon}$. Using Lemmas 3.4, 3.10 and Remark 3.3, we find that $\hat{q}_{\varepsilon}=\left(\hat{u}_{\varepsilon}, \hat{v}_{\varepsilon}\right)$ is a (time-independent) strict subsolution of (1) if $\varepsilon$ is sufficiently small; moreover we have

$$
p_{1} \gg \hat{q}_{\varepsilon} \gg p_{2} .
$$

Similarly, by making use of the function $W_{\varepsilon}$ instead of $w_{\varepsilon}$, we see that there exists a strict supersolution $\hat{\hat{q}}_{\varepsilon}=\left(\hat{\hat{u}}_{\varepsilon}, \hat{\hat{v}}_{\varepsilon}\right)$ satisfying $p_{1} \gg \hat{\hat{q}}_{\varepsilon} \gg p_{2}$ and that

$$
\hat{\hat{u}}_{\varepsilon}(x)=\bar{u}_{1}, \hat{\hat{v}}_{\varepsilon}(x)=\bar{v}_{1} \quad\left(x_{1} \geqq 0\right)
$$

for any $0<\varepsilon \leqq 1 / 2$. This, together with (32), implies $\hat{\hat{q}}_{\varepsilon} \gg \hat{q}_{\varepsilon}$; hence, by Theorem 2.6 , there exists a stable equilibrium solution $p$ of (1) satisfying $\hat{\hat{q}}_{\varepsilon} \gg p \gg \hat{q}_{\varepsilon}$ (provided that $\varepsilon$ is sufficiently small). Obviously $p$ is spatially-inhomogeneous. This completes the proof of Theorem A. 
Example 3.11. A typical example that meets the conditions in Theorem A (and Remark 3.1) is the Lotka-Volterra system

$$
\begin{cases}\frac{\partial u}{\partial t}=d_{1} \Delta u+\left(r_{1}-a_{1} u-b_{1} v\right) u & (x \in \Omega, t>0) \\ \frac{\partial v}{\partial t}=d_{2} \Delta v+\left(r_{2}-a_{2} u-b_{2} v\right) v & (x \in \Omega, t>0),\end{cases}
$$

where $d_{i}, r_{i}, a_{i}, b_{i}(i=1,2)$ are positive constants satisfying

$$
\frac{a_{1}}{a_{2}}<\frac{r_{1}}{r_{2}}<\frac{b_{1}}{b_{2}} .
$$

By virtue of the above inequalities the system

$$
\left\{\begin{array}{l}
\frac{d u}{d t}=\left(r_{1}-a_{1} u-b_{1} v\right) u \\
\frac{d v}{d t}=\left(r_{2}-a_{2} u-b_{2} v\right) v
\end{array}\right.
$$

possesses a pair of asymptotically stable equilibria

$$
\left(r_{1} / a_{1}, 0\right), \quad\left(0, r_{2} / b_{2}\right) \text {. }
$$

Thus Theorem A, together with Remark 3.1, guarantees the existence of a stable spatially-inhomogeneous equilibrium solution, which, in other words, implies the occurrence of pattern formation. Figure 2 shows a numerical experiment that illustrates how such a pattern evolves from an embrionic initial state (in the case $d_{1}=0.3, d_{2}=1.5, r_{1}=r_{2}=1, a_{1}=b_{2}=1, a_{2}=b_{1}=1.2, l=1.2$, $r_{M}=0.2$ ). From an ecological point of view, such a phenomenon can be interpreted as spatial segregation between two competing species. 

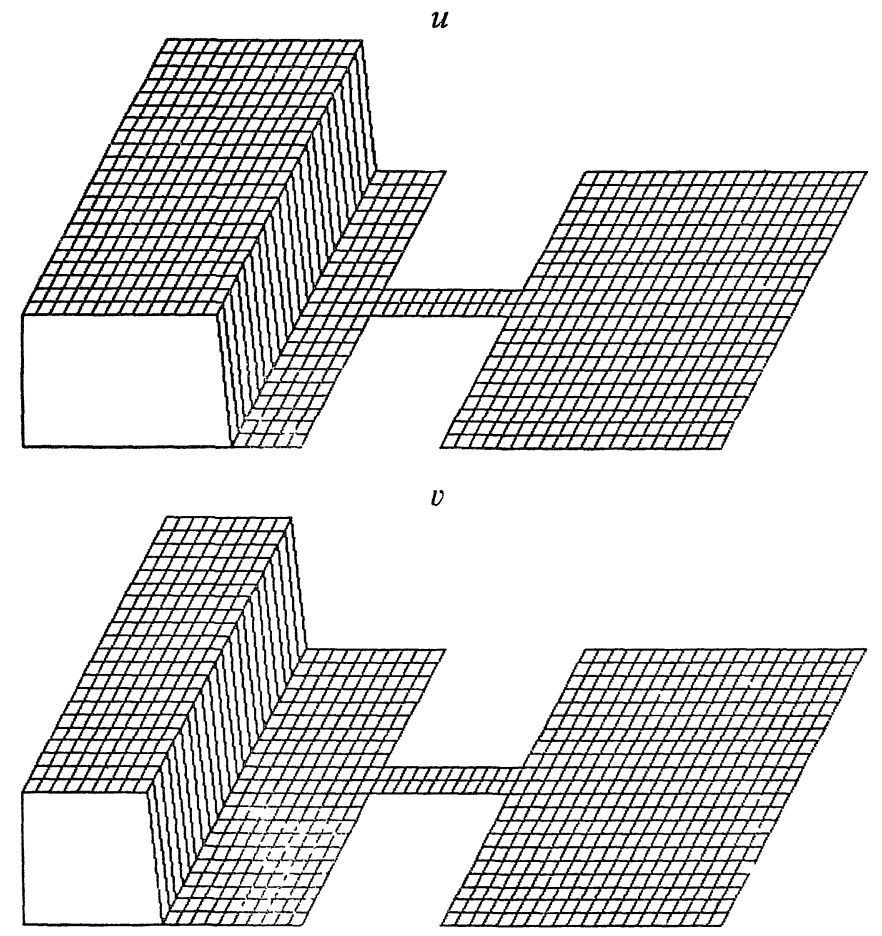

Fig. 2(a). $\quad t=0$
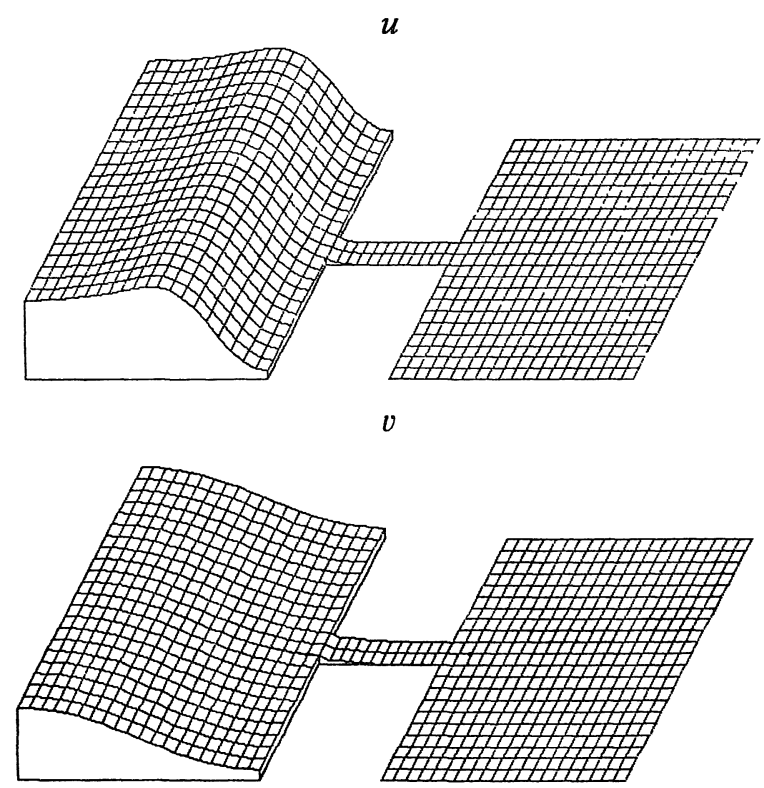

Fig. 2(b). $\quad t=0.5$ 


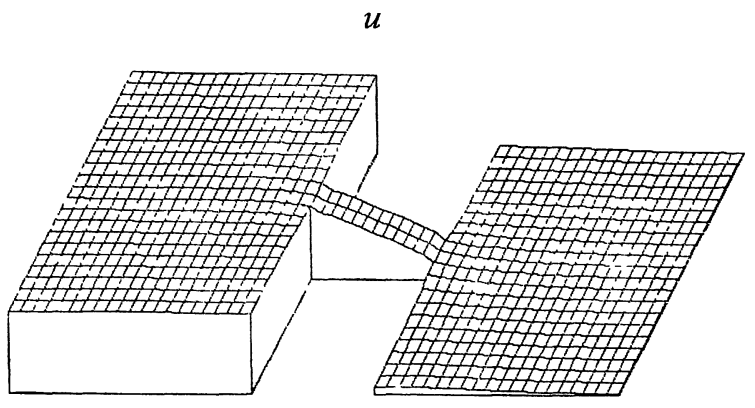

$v$

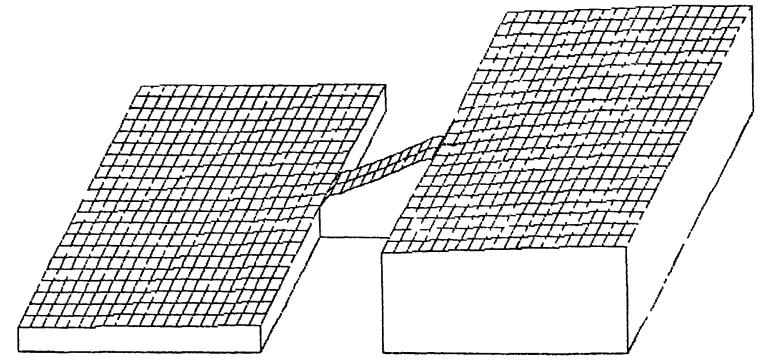

Fig. 2(c). $t=10$

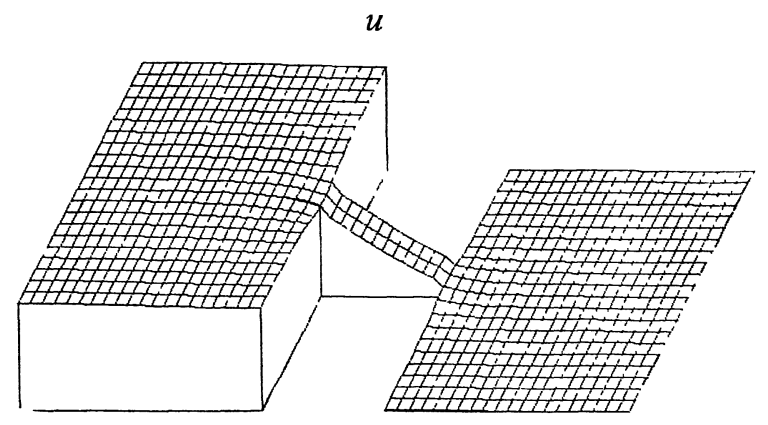

$v$

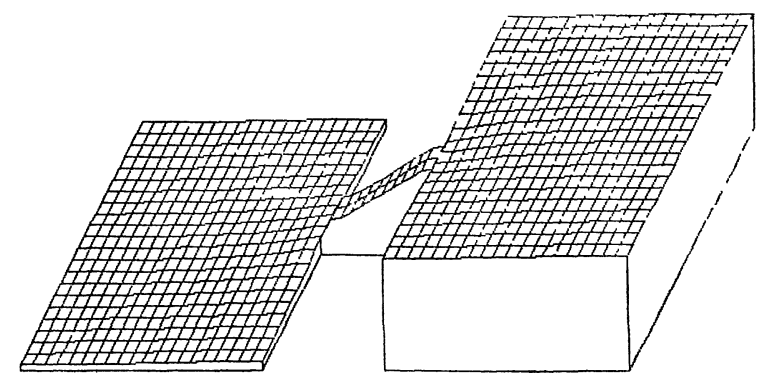

Fig. 2 (d). $\quad t \geqq 100$ 


\section{§4. Systems with Cross-Diffusion}

In this section we consider a system of equations of the form (4) under the initial and the boundary conditions (1b) (1c), where $d_{1}, d_{2}$ are positive constants, $\alpha, \beta$ are nonnegative constants, $f, g$ are functions satisfying (2), and $\Omega$ is a bounded domain in $\boldsymbol{R}^{n}$ with smooth boundary $\partial \Omega$. We assume that

$$
f(0, v) \geqq 0, g(u, 0) \geqq 0
$$

for any $u \geqq 0, v \geqq 0$. The condition (33) guarantees that any solution of (4), (1b), (1c) with $u_{0} \geqq 0, v_{0} \geqq 0$ satisfies $u \geqq 0, v \geqq 0$ for $t \geqq 0$. Hereafter we shall be concerned only with nonnegative solutions.

If $\alpha=\beta=0$, then (4) reduces to the semilinear system (1a) whose properties have already been discussed in the preceding sections. In the case $\alpha \neq 0$ and/or $\beta \neq 0$, however, the dynamical behavior of (4) is, in general, quite different from that of (1). For instance, the comparison principle no longer holds in this case.

The aim of this section is to give a bifurcation theoretical aspect of the problem of pattern formation in the case $\alpha \neq 0$ or $\beta \neq 0$. In what follows the term "stable" always refers to the linearized stability. The question on nonlinear stability still remains open.

The local existence theorem for (4), (1b), (1c) has recently been established by $\operatorname{Kim}[8]$ in the case $n=1$. In the more special case $\beta=0, n=1$, one can obtain much better a priori estimates, from which the global existence of solutions follows for suitable $f$ and $g$ to be specified later. (See Masuda and Mimura [13].) Also, in the case $\alpha=\beta>0, n=1$, the global existence has been proved in [8].

In ecological terms, the system (4) describes the time-evolution of population distribution of two species which are "biodiffusing" over the domain $\Omega$. According to Okubo [17], there are, typically, three types of biological "diffusion", that appear in the theory of population dynamics. In terms of differential equations, any of these three types can be expressed as $\partial w / \partial t+\operatorname{div} J=F$, where $w$ is the population density, $J$ is the flux and $F$ represents the population supply due to births and deaths; but the expression of $J$ differs from type to type:

(i) Fickian type - the individuals are moving fully at randam in such a way that the Fick's law holds; hence

$$
\operatorname{div} J=-\nabla\{d(x, t) \nabla w\},
$$


provided that the diffusion takes place isotropically, where $d(x, t)$ is the diffusion coefficient.

(ii) Repulsive type - the individuals are moving at randam due to repulsive forces, and we have the expression

$$
\operatorname{div} J=-\Delta\{d(x, t) w\},
$$

where $d$ is again called the diffusion coefficient; intuitively, $d$ represents the degree of uncomfortableness (or the measure of repulsive force) of each place where the individuals are located.

(iii) Attractive type - the diffusion is caused by attractive forces and

$$
\operatorname{div} J=-\nabla\left[\{d(x, t)\}^{2} \nabla\left\{\frac{w}{d(x, t)}\right\}\right] .
$$

If the diffusion coefficient $d$ is independent of $x$, then one easily finds that the above three types are equivalent; however, if $d$ is spatially-inhomogeneous, these three "diffusions" refer to rather different phenomena.

The system (4), of course, belongs to the second type, with the diffusion coefficients depending on the unknowns $u$ and $v$. More, precisely, the increase of the population density of one species causes the increase of repulsive forces against the other species.

We begin with the following proposition:

Proposition 4.1. Let $(u, v)$ be a solution of (4), (1b), (1c) that exists globally in time. Suppose there exist constants $K_{1}, K_{2}$ such that

$$
0 \leqq u(x, t) \leqq K_{1}, \quad 0 \leqq v(x, t) \leqq K_{2}
$$

for all $x \in \Omega, 0 \leqq t<+\infty$. Put

$$
\begin{gathered}
f_{1}=\max \left|\frac{\partial f}{\partial u}(u, v)\right|, \quad f_{2}=\max \left|\frac{\partial f}{\partial v}(u, v)\right|, \\
g_{1}=\max \left|\frac{\partial g}{\partial u}(u, v)\right|, \quad g_{2}=\max \left|\frac{\partial g}{\partial v}(u, v)\right|,
\end{gathered}
$$

where the $\max$ is taken over the region $0 \leqq u \leqq K_{1}, 0 \leqq v \leqq K_{2}$, and, finally, denote by $\lambda$ the second (i.e. the least positive) eigenvalue of $-\Delta$ on $\Omega$ under the homogeneous Neumann boundary conditions. If

$$
\left\{\begin{array}{l}
d_{1}>\frac{f_{1}}{\lambda}, \quad d_{2}>\frac{g_{2}}{\lambda} \\
\left(d_{1}-\frac{f_{1}}{\lambda}\right)\left(d_{2}-\frac{g_{2}}{\lambda}\right) \geqq\left(\alpha K_{1}+\frac{f_{2}}{\lambda}\right)\left(\beta K_{2}+\frac{g_{1}}{\lambda}\right),
\end{array}\right.
$$


then

$$
\int_{\Omega}\left[\{u(x, t)-\bar{u}(t)\}^{2}+\{v(x, t)-\bar{v}(t)\}^{2}\right] d x \longrightarrow 0
$$

exponentially as $t \rightarrow+\infty$, where

$$
\bar{u}=\frac{1}{|\Omega|} \int u d x, \quad \bar{v}=\frac{1}{|\Omega|} \int v d x .
$$

Proof. The following argument is along the lines found in [3]. Set

$$
J_{1}(t)=\frac{1}{2} \int_{\Omega}\{u(x, t)-\bar{u}(t)\}^{2} d x .
$$

Using Green's formula, we get

$$
\begin{aligned}
\frac{d}{d t} J_{1}(t) & =\int_{\Omega}\{u-\bar{u}\}\left\{\frac{\partial u}{\partial t}-\frac{d \bar{u}}{d t}\right\} d x \\
& =-\int_{\Omega}\left\{\left(d_{1}+\alpha v\right)|\nabla u|^{2}+\alpha u \nabla u \cdot \nabla v-(u-\bar{u})(f-\bar{f})\right\} d x
\end{aligned}
$$

where

$$
\bar{f}(t)=\frac{1}{|\Omega|} \int_{\Omega} f(u(x, t), v(x, t)) d x
$$

Recall that

$$
\int_{\Omega}|\nabla w|^{2} d x \geqq \lambda \int_{\Omega} w^{2} d x
$$

holds for any $w \in H^{1}(\Omega)$ satisfying

$$
\int_{\Omega} w d x=0
$$

Applying the above inequality to $u-\bar{u}$ and $f-\bar{f}$ yields

$$
\begin{aligned}
\frac{d}{d t} J_{1}(t) & \leqq-d_{1}\|\nabla u\|^{2}+\alpha K_{1}\|\nabla u\|\|\nabla v\|+\frac{1}{\lambda}\|\nabla u\|\|\nabla f\| \\
& \leqq-d_{1}\|\nabla u\|^{2}+\alpha K_{1}\|\nabla u\|\|\nabla v\|+\frac{1}{\lambda}\|\nabla u\| \times \\
& \left(f_{1}\|\nabla u\|+f_{2}\|\nabla v\|\right),
\end{aligned}
$$

where

$$
\|w\|^{2}=\int_{\Omega} w^{2} d x
$$

Similarly, letting

$$
J_{2}(t)=\frac{1}{2} \int_{\Omega}\{v(x, t)-\bar{v}(t)\}^{2} d x,
$$


we get

$$
\begin{gathered}
\frac{d}{d t} J_{2}(t) \leqq-d_{2}\|\nabla v\|^{2}+\beta K_{2}\|\nabla u\|\|\nabla v\|+\frac{1}{\lambda}\|v\| \times \\
\left(g_{1}\|\nabla u\|+g_{2}\|\nabla v\|\right) .
\end{gathered}
$$

Combining (36) and (37), and applying (35) again, we see that the conclusion of Proposition 4.1 holds.

Corollary 4.2. Let $K_{1}, K_{2}$ be any positive numbers and let $f_{i}, g_{i}(i=1,2)$ be as in (33). Suppose (34) holds. Then any solution $(u, v)$ to

$$
\begin{cases}\Delta\left\{\left(d_{1}+\alpha v\right) u\right\}+f(u, v)=0 & (x \in \Omega) \\ \Delta\left\{\left(d_{2}+\beta u\right) v\right\}+g(u, v)=0 & (x \in \Omega) \\ \frac{\partial u}{\partial n}=\frac{\partial v}{\partial n}=0 & (x \in \partial \Omega)\end{cases}
$$

satisfying

$$
0 \leqq u(x) \leqq K_{1}, \quad 0 \leqq v(x) \leqq K_{2} \quad \text { in } \quad \Omega
$$

is spatially-homogeneous (i.e. constant).

In what follows we shall consider a more specific case where

$$
\left\{\begin{array}{l}
f(u, v)=\left(r_{1}-a_{1} u-b_{1} v\right) u \\
g(u, v)=\left(r_{2}-a_{2} u-b_{2} v\right) v .
\end{array}\right.
$$

Here $r_{i}, a_{i}, b_{i}(i=1,2)$ are positive constants satisfying

$$
\frac{a_{1}}{a_{2}}>\frac{r_{1}}{r_{2}}>\frac{b_{1}}{b_{2}} \text {. }
$$

The condition (40) implies that the ODE system (10) with (39) possesses a unique equilibrium point $\left(u^{*}, v^{*}\right)$ in the region $u>0, v>0$, more precisely

$$
u^{*}=\frac{r_{1} b_{2}-r_{2} b_{1}}{a_{1} b_{2}-a_{2} b_{1}}, \quad v^{*}=\frac{a_{1} r_{2}-a_{2} r_{1}}{a_{1} b_{2}-a_{2} b_{1}}
$$

and that $\left(u^{*}, v^{*}\right)$ is globally asymptotically stable - that is, the stable manifold of $\left(u^{*}, v^{*}\right)$ is the whole region $u>0, v>0$.

In the case (39), the statement of Corollary 4.2 can be improved as follows:

Lemma 4.3. Let $K_{1}, K_{2}$ be positive constants satisfying

$$
d_{1} d_{2} \geqq \frac{\left(a_{2} u^{*} K_{2}+b_{1} v^{*} K_{1}\right)^{2}}{4 a_{2} b_{1} u^{*} v^{*}} .
$$


Then, other than $\left(u^{*}, v^{*}\right)$, there exists no solution to (38), (39) that is contained in the region $0<u \leqq K_{1}, 0<v \leqq K_{2}$.

Proof. Let $(u(x, t), v(x, t))$ be a solution to (4), (1b) (1c), (39) that exists globally in time. Set

$$
E(t)=\int_{\Omega}\left\{a_{2}\left(u-u^{*}-u^{*} \log \frac{u}{u^{*}}\right)+b_{2}\left(v-v^{*}-v^{*} \log \frac{v}{v^{*}}\right)\right\} d x .
$$

A simple calculation shows that

$$
\begin{aligned}
& \frac{d}{d t} E=-\int_{\Omega}\left(A|\nabla u|^{2}+B \nabla u \cdot \nabla v+C|\nabla v|^{2}\right) d x- \\
& \quad-\int_{\Omega}\left\{a_{1} a_{2}\left(u-u^{*}\right)^{2}+2 a_{2} b_{1}\left(u-u^{*}\right)\left(v-v^{*}\right)+b_{1} b_{2}\left(v-v^{*}\right)^{2}\right\} d x,
\end{aligned}
$$

where

$$
\begin{aligned}
& A=a_{2}\left(d_{1}+\alpha v\right) \frac{u^{*}}{u^{2}}, \quad B=\alpha a_{2} \frac{u^{*}}{u}+\beta b_{1} \frac{v^{*}}{v}, \\
& C=b_{1}\left(d_{2}+\beta u\right) \frac{v^{*}}{v^{2}} .
\end{aligned}
$$

Since the second integral on the right-hand side of (42) is always nonnegative by the condition (40), we have

$$
\frac{d}{d t} E \leqq 0
$$

provided that $0<u(x, t) \leqq K_{1}, 0<v(x, t) \leqq K_{2}$ for all $x \in \Omega, t \geqq 0$ and that (41) holds. The equality in (43) holds if and only if $u \equiv u^{*}, v \equiv v^{*}$. The conclusion of Lemma 4.3 now follows immediately.

Remark 4.4. Since $E$ attains the minimal value 0 only when $u \equiv u^{*}, v \equiv v^{*}$, it is clear from the proof of Lemma 4.3 that any solution $(u, v)$ to (4), (1b), (1c), (39) that exists globally in time and satisfies $0<u(x, t) \leqq K_{1}, 0<v(x, t) \leqq K_{2}$ for all $x \in \Omega, t \geqq 0$ will converge to $\left(u^{*}, v^{*}\right)$ in $L^{p}(\Omega)$ (for any $1 \leqq p<\infty$ ) as $t \rightarrow+\infty$, provided that the inequality (41) holds. This fact strongly suggests that $\left(u^{*}, v^{*}\right)$ is asymptotically stable as an equilibrium solution to (4), (1b), (1c), (39), though the rigorous proof of the stability requires further a priori estimates and a global existence theorem, which are still open questions. Note, however, that the stability of $\left(u^{*}, v^{*}\right)$ follows immediately in some special cases (such as the case $n=1, \alpha \beta=0$ or the case $n=1, \alpha=\beta$ ) where sufficient a priori estimates along with the global existence theorem have already been established by [13] and [8]. 
In what follows we consider the case $\beta=0$ for simplicity, namely

$$
\begin{cases}\frac{\partial u}{\partial t}=\Delta\left\{\left(d_{1}+\alpha v\right) u\right\}+\left(r_{1}-a_{1} u-b_{1} v\right) u & (x \in \Omega, t>0) \\ \frac{\partial v}{\partial t}=d_{2} \Delta v+\left(r_{2}-a_{2} u-b_{2} v\right) v & (x \in \Omega, t>0) \\ u(x, 0)=u_{0}(x), v(x, 0)=v_{0}(x) & (x \in \Omega) \\ \frac{\partial u}{\partial n}=\frac{\partial v}{\partial n}=0 & (x \in \partial \Omega, t>0) .\end{cases}
$$

As is mentioned above, the global existence theorem for this system is well established in [13] in the case $n=1$. The stationary problem associated with (44) is

$$
\begin{cases}\Delta\left\{\left(d_{1}+\alpha v\right) u\right\}+\left(r_{1}-a_{1} u-b_{1} v\right) u=0 & (x \in \Omega) \\ d_{2} \Delta v \quad+\left(r_{2}-a_{2} u-b_{2} v\right) v=0 & (x \in \Omega) \\ \frac{\partial u}{\partial n}=\frac{\partial v}{\partial n}=0 & (x \in \partial \Omega)\end{cases}
$$

Putting $w=\left(1+\alpha v / d_{1}\right) u$ and applying the maximum principle to the pair $w, v$, we see that any nonnegative solution to (45) satisfies

$$
\left\{\begin{array}{l}
0 \leqq u(x) \leqq \frac{r_{1}}{a_{1}}\left(1+\frac{\alpha r_{2}}{d_{1} b_{2}}\right) \\
0 \leqq v(x) \leqq \frac{r_{2}}{b_{2}}
\end{array}\right.
$$

Combining this and Lemma 4.3, we get

Corollary 4.5. Assume (40) holds and that

$$
d_{1} d_{2} \geqq \frac{\left\{\frac{r_{2} a_{2}}{b_{2}} u^{*}+\frac{r_{1} b_{1}}{a_{1}}\left(1+\frac{\alpha r_{2}}{d_{1} b_{2}}\right)\right\}^{2}}{4 a_{2} b_{1} u^{*} v^{*}} .
$$

Then (45) possesses only constant solutions in the region $u \geqq 0, v \geqq 0$; namely

$$
\left(u^{*}, v^{*}\right),\left(0, \frac{r_{2}}{b_{2}}\right),\left(\frac{r_{1}}{a_{1}}, 0\right),(0,0) .
$$

The above corollary will be useful in the later discussion.

In order to find a stable spatially-inhomogeneous solution to (45), we shall regard $d_{1}, d_{2}$ or $\alpha$ as bifurcation parameters and study the bifurcation diagram from a global aspect. The spatially-homogeneous solutions $\left(u^{*}, v^{*}\right),\left(0, r_{2} / b_{2}\right)$, $\left(r_{1} / a_{1}, 0\right),(0,0)$ are henceforth regarded as trivial solutions. One can easily 
show that no bifurcation occurs either from $\left(0, r_{2} / b_{2}\right),\left(r_{1} / a_{1}, 0\right)$ or $(0,0)$. Let us therefore study the bifurcation from the point $\left(u^{*}, v^{*}\right)$.

The linearized stability of $\left(u^{*}, v^{*}\right)$ as an equilibrium solution to (44) is determined by the sign of the least eigenvalue of the following eigenvalue problem associated with the linearized problem for (45):

$$
\begin{cases}\left(d_{1}+\alpha v^{*}\right) \Delta \phi+\alpha u^{*} \Delta \psi-a_{1} u^{*} \phi-b_{1} u^{*} \psi=-\mu \phi & (x \in \Omega) \\ d_{2} \Delta \psi-a_{2} v^{*} \phi-b_{2} v^{*} \psi=-\mu \psi & (x \in \Omega) \\ \frac{\partial \phi}{\partial n}=\frac{\partial \psi}{\partial n}=0 & (x \in \partial \Omega) .\end{cases}
$$

If the least eigenvalue of (47) is positive, then $\left(u^{*}, v^{*}\right)$ is said to be linearly stable; if it is negative, then $\left(u^{*}, v^{*}\right)$ is linearly unstable. Let $\lambda_{k}, k=0,1,2, \ldots$, be the $(k+1)$-th eigenvalue of $-\Delta$ on $\Omega$ under the homogeneous Neumann boundary conditions, and let $\varphi_{k}$ be the corresponding eigenfunction. Using the eigenfunction expansion of $\phi, \psi$ in terms of $\left\{\varphi_{k}\right\}$, we easily find that $\mu$ is an eigenvalue of (47) if and only if

$$
\operatorname{det}\left(\begin{array}{cc}
\left(d_{1}+\alpha v^{*}\right) \lambda_{k}+a_{1} u^{*}-\mu & \alpha u^{*} \lambda_{k}+b_{1} u^{*} \\
a_{2} v^{*} & d_{2} \lambda_{k}+b_{2} v^{*}-\mu
\end{array}\right)=0
$$

for some nonnegative integer $k$. In particular, $\mu=0$ is an eigenvalue of (47) if and only if

$$
\begin{aligned}
\left(d_{1}+v^{*}\right) d_{2} \lambda_{k}{ }^{2} & +\left\{b_{2} v^{*} d_{1}+a_{1} u^{*} d_{2}+\alpha\left(b_{2} v^{*}-a_{2}\right) v^{*}\right\} \lambda_{k} \\
& +\left(a_{1} b_{2}-a_{2} b_{1}\right) u^{*} v^{*}=0
\end{aligned}
$$

for some $k$. Note that (49) does not hold when $k=0$, because of (40) and $\lambda_{0}=0$. In the case $n=1, \Omega=(0,1)$, we have $\lambda_{k}=k^{2} \pi^{2}$. Fig. 3 illustrates the family of curves $\left\{C_{k}\right\}_{k=1}^{\infty}$ (in $(d, \alpha)$-plane where $d=d_{1}=d_{2}$ ) on which (49) is satisfied (here $r_{i}, a_{i}, b_{i} i=1,2$, are fixed). It is not difficult to check that each point on $C_{k}, k=$ $1,2, \ldots$, is a bifurcation point, where the term "bifurcation" refers to that from $p^{*}=\left(u^{*}, v^{*}\right)$. Of course the bifurcating solutions are spatially-inhomogeneous.

Let us now fix $\alpha>0$ and take $d=d_{1}=d_{2}$ to be the only bifurcation parameter, which varies over the interval $(0,+\infty)$. Hereafter, by a "solution" to the system (44), we mean a triple $(u, v, d)$. The set of all solutions forms a subset of $H_{N}^{2}(0,1)$ $\times H_{N}^{2}(0,1) \times(0,+\infty)$, where $H_{N}^{2}(0,1)$ denotes the closed linear hull of $\{\cos k \pi x\}_{k=0}^{\infty}$ in the Sobolev space $H^{2}(0,1)$.

The following is the main theorem of this section. 


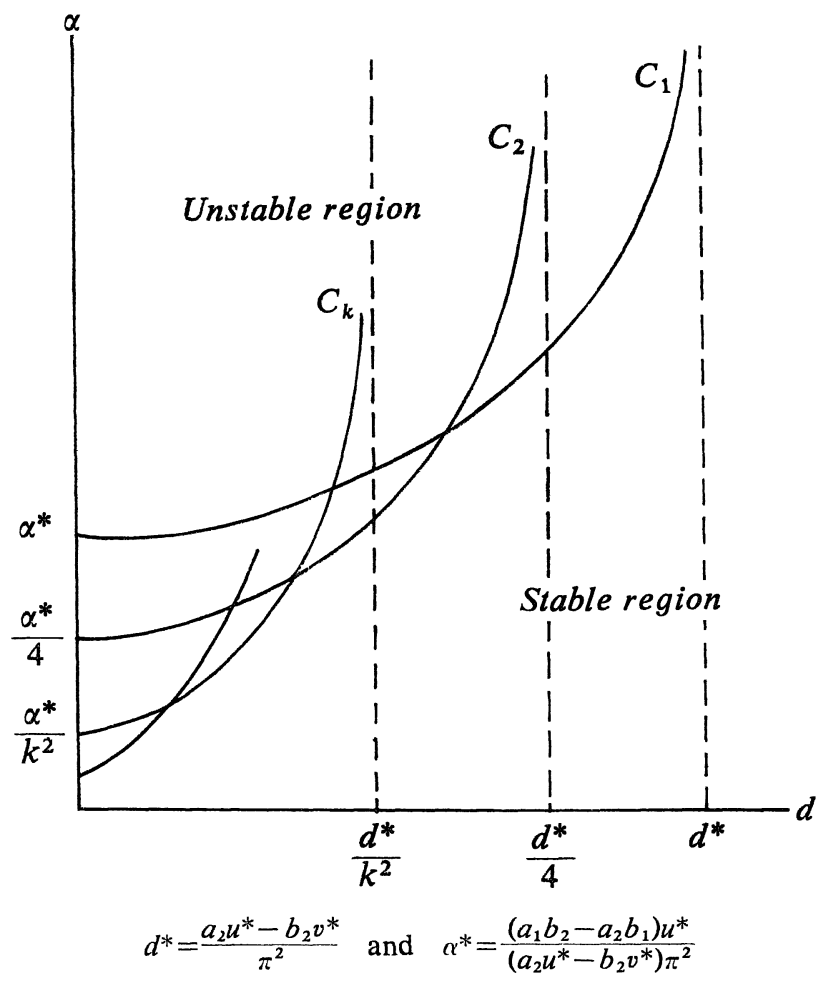

Fig. 3. Bifurcation curves in $(d, \alpha)$ plane $(n=1)$.

Theorem B. Let $n=1, \Omega=(0,1)$, and let $S$ denote the set of all solutions to (45). Also let $S_{0}$ be the set of all trivial (i.e., spatially-homogeneous) solutions. Suppose $\alpha>0$ is such that the half line $l_{\alpha}=\{(d, \alpha) \mid d>0\}$ does not pass through any intersection point of the curves $C_{k}, k=1,2, \ldots$. Denote by $\left(d^{(k)}, \alpha\right)$ the intersection point of $l_{\alpha}$ and $C_{k}$ (if they ever intersect) and let $\left(d^{\left(k_{0}\right)}, \alpha\right)$ be the rightmost one among the points $\left(d^{(k)}, \alpha\right), k=1,2, \ldots$. Finally, denote by $S_{k_{0}}$ the connected component of $\overline{S \backslash S_{0}}$ containing $\left(u^{*}, v^{*}, d^{\left(k_{0}\right)}\right)$. Then there exists a $d_{c} \geqq d^{\left(k_{0}\right)}$ such that

$$
\mathfrak{P} S_{k_{0}}=\left(0, d_{c}\right],
$$

where $\mathfrak{P}: H_{N}^{2}(0,1) \times H_{N}^{2}(0,1) \times(0,+\infty) \rightarrow(0,+\infty)$ is a projection map.

Proof. This theorem is a vector-valued version of Theorem 2.5 in [2], which is on scalar equations. Since the argument in [20] does not apply to the vector case, we need quite a different approach. The following argument follows partly the line found in Fujii, Mimura and Nishiura [4]. 
First of all, it is clear from (49) that

$$
(d, \alpha) \in C_{k} \Longleftrightarrow\left(k^{2} d, k^{2} \alpha\right) \in C_{1}
$$

for any $k=1,2, \ldots$ Let

$$
C_{1} \cap C_{2}=\left\{P_{1}\right\}
$$

and let $\tilde{C}_{1}$ be the subset of $C_{1}$ lying on the right of $P_{1}$. We shall first prove that the conclusion of Theorem B holds if $\left(d^{\left(k_{0}\right)}, \alpha\right)$ lines on $\widetilde{C}_{1}$ (hence $k_{0}=1$ in that case).

By virtue of Corollary $4.5, \mathfrak{B S} S_{1}$ is bounded from above; hence either

$$
\mathfrak{P S} S_{1}=\left[\delta, d_{c}\right]
$$

for some $0<\delta \leqq d_{c}$ or

$$
\mathfrak{P S}=\left(0, d_{c}\right]
$$

for some $d_{c}>0$. Assuming that (51a) holds, we shall derive a contradiction.

Let $\mathfrak{Q}=I-\mathfrak{P}$, i.e.,

$$
\mathfrak{Q}: H_{N}^{2}(0,1) \times H_{N}^{2}(0,1) \times(0,+\infty) \longrightarrow H_{N}^{2}(0,1) \times H_{N}^{2}(0,1)
$$

be a projection map. In view of (46) and (51a), and applying the usual a priori estimates to the pair $w=\left(1+\alpha v / d_{1}\right) u$, $v$, we see that $S_{1}$ is bounded in $H_{N}^{2}(0,1)$ $\times H_{N}^{2}(0,1)$. Combining this and (51a), we find that $S_{1}$ is bounded (actually, compact) in $H_{N}^{2}(0,1) \times H_{N}^{2}(0,1) \times(0,+\infty)$. Consequently, as $\left(u^{*}, v^{*}, d^{(1)}\right)$ is a simple bifurcation point, it follows from the theorem of Rabinowitz [20; Theorem 1.3] that

$$
\left(u^{*}, v^{*}, d^{(k)}\right) \in S_{1}
$$

for some $k \geqq 2$. Let $k$ be the largest integer that satisfies (52). Since (50) holds by virtue of (49), we have

$$
\left(\bar{k}^{2} d^{(\bar{k})}, \bar{k}^{2} \alpha\right) \in \widetilde{C}_{1} .
$$

Denote by $\hat{S}_{1}$ the connected component of $\overline{S \mid \hat{S}_{0}}$, containing $\left(u^{*}, v^{*}, \bar{k}^{2} d^{(\bar{k})}\right)$, where $\hat{S}$ is the set of all solutions to (47), with $\alpha$ being replaced by $\bar{k}^{2} \alpha$. Applying Rabinowitz's theorem to $\hat{S}_{1}$, we obtain the following alternatives: (i) $\hat{S}_{1}=$ $(0, \bar{d}]$ for some $\bar{d}>0$ or else (ii) $\hat{S}_{1}$ contains a point $\left(u^{*}, v^{*}, d^{\prime}\right)$, where $d^{\prime}$ is such that $\left(d^{\prime}, \bar{k}^{2} \alpha\right) \in \widetilde{C}_{\overline{\bar{k}}}$ for some $\overline{\bar{k}} \geqq 2$. Considering that the bifurcation branch occurring from $\left(\bar{k}^{2} d^{(\bar{k})}, \bar{k}^{2} \alpha\right) \in \widetilde{C}_{1}$ can be embedded in a natural way into the bifurcation branch occurring from $\left(d^{(\bar{k})}, \alpha\right) \in C_{\bar{k}}$ through the transformation 


$$
\left\{\begin{array}{l}
\left(\bar{u}(x), \bar{v}(x), \bar{k}^{2} d\right) \longrightarrow(u(x), v(x), d) \\
\bar{k}^{2} \alpha \longrightarrow \alpha,
\end{array}\right.
$$

where $u, v$ are obtained by reflecting the functions $\bar{u}(\bar{k} x), \bar{v}(\bar{k} x)(0 \leqq x \leqq 1 / \bar{k})$ successively over the interval $0 \leqq x \leqq 1$, we find that the above alternative (i) implies that the bifurcation branch from $\left(u^{*}, v^{*}, d^{(\bar{k})}\right)$ can be continued down to $d=0$, hence so can be $S_{1}$, contradicting the supposition that (51a) holds. Similarly, the alternative (ii) implies that the bifurcation branch from $\left(u^{*}, v^{*}, d^{(k)}\right)$ contains the point $\left(u^{*}, v^{*}, d^{(\bar{k})}\right)$, hence so does $S_{1}$, contradicting the supposition that $\bar{k}$ is the maximal value of $k$ for which (52) holds. These contradictions show that the supposition (51a) is false, hence the conclusion of Theorem B holds in the special case $\left(d^{\left(k_{0}\right)}, \alpha\right) \in \widetilde{C}_{1}$. Using the transformation (53) again, we see that the conclusion of Theorem B also holds if $\left(d^{\left(k_{0}\right)}, \alpha\right) \in \widetilde{C}_{k_{0}}$, where $\widetilde{C}_{k_{0}}$ is the set of points of $C_{k_{0}}$ lying on the right of $P_{k_{0}}$, with $P_{k_{0}}$ being the intersection point of the curves $C_{k_{0}}, C_{2 k_{0}}$. Combining these, we get to the completion of the proof of Theorem B.

Remark 4.6. As illustrated in Figure 3, $\left(u^{*}, v^{*}\right)$ is stable when $d>d^{\left(k_{0}\right)}$ and is unstable when $d<d^{\left(k_{0}\right)}$. If the bifurcation occurs supercritically, then,

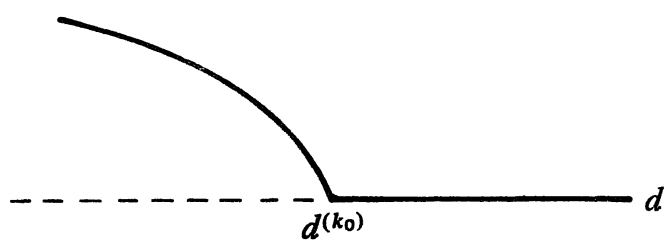

Supercritical bifurcation

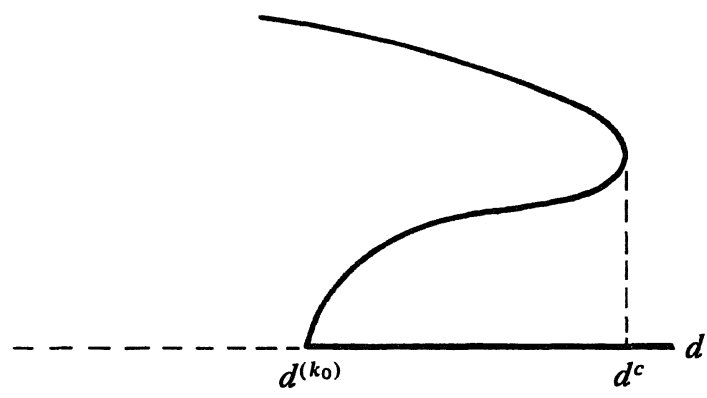

Subcritical bifurcation

Fig. 4. 
as is well known, the bifurcating solutions are stable near the bifurcation point. (Here, as usual, we are varying the bifurcation parameter from the stable region toward the unstable region; hence, in the present problem, "super-" refers to the direction along which $d$ is decreasing.) On the other hand, if the bifurcation is subcritical, then the bifurcating solutions are unstable near the bifurcation point. Under certain circumstances, both types of bifurcation can occur in our problem. However, Theorem B implies that, even in the case of subcritical bifurcation, the bifurcation branch should eventually turn to the left, thereby, possibly, gaining stability (see Figure 4). Whether the branch after the turning point is really stable or not still remains to be an open question (cf. Knightly and Sather [12] for related questions), but it is numerically confirmed that the answer is yes in the present problem. Moreover, our numerical experiments further indicate that the stability of the bifurcation branch will be maintained down to the point $d=0$ (possibly, after exchanging stability with secondary or tertiary branches and so forth; cf. [4; Figures 4.2, 4.9]).

\section{References}

[1] Brown, P., Decay to uniform states in competitive systems, preprint, (1981).

[2] Casten, R. G. and Holland, C. J., Instability results for reaction diffusion equations with Neumann boundary conditions, J. Differential Equations, 27 (1978), 266-273.

[ 3 ] Conway, E., Hoff, D. and Smoller, J., Large time behavior of solutions of systems of nonlinear reaction-diffusion equations, SIAM J. Appl. Math., 35 (1978), 1-16.

[4] Fujii, H., Mimura, M. and Nishiura, Y., A picture of the global bifurcation diagram in ecological interacting and diffusing systems, Physica D, 5D (1982), 1-42.

[5] Hale, J. K. and Vegas, J., A nonlinear parabolic equation with varying domain, LCDS Report 81-1, Division of Appl. Math., Brown Univ. (1980).

[6] Hirsch, M. W., Differential equations and convergence almost everywhere of strongly monotone semiflows, PAM Technical Report, Univ. Calif. Berkeley (1982).

[7] Keyfitz, B. L. and Kuiper, H. J., Bifurcation resulting from changes in domain in a reaction-diffusion equation, to appear in J. Differential Equations.

[8] Kim, J. U., Smooth solutions to a quasi-linear system of diffusion equations for a certain population model, MRC Technical Summary Report, Univ. Wisconsin (1982).

[9] Kishimoto, K., Instability of non-constant equilibrium solutions of a system of competition-diffusion equations, J. Math. Biol., 13 (1981) 105-114.

[10] Kishimoto, K., Mimura, M. and Yoshida, K., Stable spatio-temporal oscillations of diffusive Lotka-Volterra systems with three or more species, to appear in J. Math. Biol.

[11] Kishimoto, K. and Weinberger, H. F., The spatial homogeneity of stable equilibria of some reaction-diffusion systems on convex domains, to appear in J. Differential Equations.

[12] Knightly, G. H. and Sather, D., Selection principles and stable subcritical solutions, preprint. 
[13] Masuda, K. and Mimura, M., On the existence of global solutions of density-dependent interaction-diffusion equations in ecology (in preparation).

[14] Matano, H., Asymptotic behavior and stability of solutions of semilinear diffusion equations, Publ. RIMS Kyoto Univ., 15 (1979), 401-454.

[15] —- Existence of nontrivial unstable sets for equilibriums of strongly orderpreserving systems, to appear in J. Fac. Sci. Univ. Tokyo.

[16] Mimura, M., Stationary pattern of some density-dependent diffusion system with competitive dynamics, Hiroshima Math. J., 11 (1981), 621-635.

[17] Okubo, A., Diffusion and ecological problems: Mathematical models, Biomath. 10 (Springer-Verlag, Berlin, Heidelberg, New York, 1980).

[18] de Mottoni, P., Qualitative analysis for some quasilinear parabolic systems, Institute of Math., Polish Academy Sci., zam. 11/79, 190 (1979).

[19] Protter, M. H. and Weinberger, H. F., Maximum principles in differential equations, (prentice-Hall, 1967).

[20] Rabinowitz, P. H., Some global results for nonlinear eigenvalue problems, J. Funct. Anal., 7 (1971), 487-513.

[21] Sattinger, D. H., Monotone methods in nonlinear elliptic and parabolic boundary value problems, Indiana Univ. Math. J., 21 (1972), 979-1000.

[22] Serrin, J., A symmetry problem in potential theory, Arch. Rational Mech. Anal., 43 (1971), 304-318.

[23] Shigesada, N., Kawasaki, K. and Teramoto, E., Spatial segregation of interacting species, J. Theor. Biol., 79 (1979), 89-99.

[24] Vegas, J., Bifurcations caused by perturbing the domain in an elliptic equation, to appear in Arch. Rational Mech. Anal. 
